\title{
Learning the Scene Illumination for Color-Based People Tracking in Dynamic Environment
}

\author{
Sinan Mutlu ${ }^{1,2}$, Tao $\mathrm{Hu}^{1,2}$, and Oswald Lanz ${ }^{1, \star}$ \\ 1 FBK Fondazione Bruno Kessler, Via Sommarive 18, I-38123 Povo(TN), Italy \\ 2 ICT Doctoral School, University of Trento, I-38123 Povo(TN), Italy \\ \{mutlu, hutao, lanz\}@fbk.eu
}

\begin{abstract}
People tracking under non-uniform illumination is challenging, as observed appearance may change as they move around in the environment. Appearance model adaptation is inconvenient over the long run as it is subject to drift, while filtering illumination information in the data through built-in invariance is sub-optimal in terms of discriminative capability. In this work, we are interested in modeling the spatial and temporal dimensions of appearance variation induced by non-uniform illumination, and to learn and adapt related parameters over time by using walking people as illumination probes. We propose a hybrid graphical model and a new message passing scheme that sequentially updates parameters of the model, so that scene illumination can be learnt online and used for robust tracking in dynamic environment.
\end{abstract}

Keywords: tracking, online learning, belief propagation, message passing, particle filter, illumination.

\section{Introduction}

People tracking is a largely studied topic in computer vision with applications in numerous contexts, including non-invasive monitoring for security and surveillance, ambient assisted living, sports analysis, etc. Despite significant progress [1, real time solutions that operate autononously in 24/7 mode show unsatisfactory performance when deployed under realistic conditions, notably, when tracking in environments with non-uniform illumination conditions that also vary over time. In this paper we address the problem of multi-camera colorbased people tracking under such assumptions. We use observations of moving people to estimate the illumination conditions of the scene as they vary across space and over time, and show how to integrate such information in a particle filter for improved color based tracking under non-uniform and changing illumination.

Most research on adaptive tracking has focussed on background updating [2] and/or adaptive appearance modelling [34. Typically, both problem settings are

\footnotetext{
* Work partially supported by EIT ICT Labs SSP 12205 Activity TIK - The Interaction Toolkit, task ID T1320A.
} 

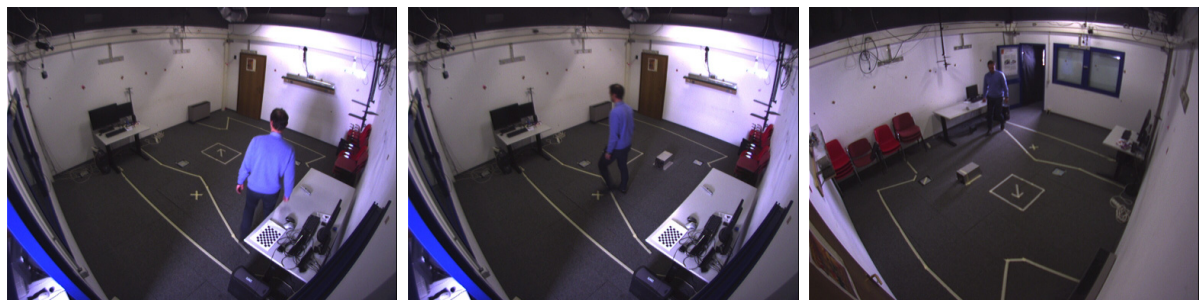

Fig. 1. Under non-uniform illumination, the appearance pattern of a target (a) may change in space (b) and with relative viewpoint (c). (All figures best viewed in color).

proper to single camera tracking, while only a few works attempt to integrate information from multiple cameras for consistent adaptation [56]. A somewhat different approach is proposed in [7, where illumination maps are introduced for multi-camera 3D tracking to encode spatial variability in unevenly illuminated scenes. Temporal variability is hereby not accounted for, and an extension is needed for robust long term monitoring in realistic environment. A quite different approach is pursued in [8] where authors propose to explicitly model light sources (the sun) and to track their positions through MCMC particle filtering for object detection in outdoor scenes. Modeling lights sources in complex indoor environments may be very costly or even impractical.

The approach in this paper builds upon the observation that colorimetric properties of clothing and skin/hair tone do not change, neither with subject position, nor over time. Instead, it is the image formation process that induces photometric distortions in their measurements as captured by cameras, resulting in appearance patterns that vary with spatial position and relative viewpoint, as a function of ambient illumination that may also change over time. In this work, we explicitly account for such distortion in color based people tracking with multiple cameras similarly to [7, and develop a novel flexible model with associated online inference to initialize and update model parameters using appearance patterns of people moving around in the scene as illumination probes.

\section{Probabilistic Tracking with Known Scene Illumination}

Our goal is to compute the 2D ground positions of people as they move around in an environment monitored by multiple calibrated cameras. We adopt a sequential Bayesian framework: given observations $z_{1: t}$, the posterior $p\left(x_{t} \mid z_{1: t}\right)$ over ground hypotheses $x_{t}$ at time $t$ is recursively computed from its previous estimate $p\left(x_{t} \mid z_{1: t}\right) \propto f\left(z_{t} \mid x_{t}\right) \int q\left(x_{t} \mid x_{t-1}\right) p\left(x_{t-1} \mid z_{1: t-1}\right) d x$ using a dynamical model $q$ and a likelihood function $f$. While $q$ may be simple (gaussian in our case), $f$ is typically highly non-linear, and no closed-form solution is found for updating $p$. Instead, a particle filter is implemented to propagate samples of $p$, and the sample means are taken to output $2 \mathrm{D}$ ground positions.

The most decisive component in the Bayesian model is the data likelihood $f$. Intuitively, $f(z \mid x)$ expresses how well a hypothesis $x$ is explained with the 
given data $z$, and should therefore be designed so as to peak when $x$ is the true hypothesis that generated $z$. And this should ideally hold true regardless of measurement conditions, in our case, illumination conditions. A possibility is to design $f$ upon invariant features, e.g. by using edges or motion, or by applying a color space transform to eliminate the illumination component in the data. Built-in invariance, however, suppresses information present in the data, resulting in sub-optimal solutions in terms of discriminative capability. In particular, ambiguity is increased in the invariant feature space, and tracking is rendered more challenging, especially with multiple people where data association and partial observation under occlusions add additional levels of difficulty.

Rather than imposing invariance by design, in this work we are interested in modeling the spatial and temporal dimensions of appearance variation induced by non-uniform illumination, to yield a likelihood for improved color-based tracking. Our assumption is that appearance patterns of a target are transformed versions of a reference template $z_{r}$ (ideally, describing target's colorimetric appearance), and that this transformation can be well approximated by a parametric generative model $\mathcal{T}(z \mid x, \lambda)$ once the pattern configuration $x$ is known. The role of $\mathcal{T}$ hereby is to remap observed patterns $z$ into the undistorted appearance space for matching with the reference template $z_{r}$, thus compensating for what we call photometric distortions.

Within this framework the scene illumination is said to be known if the parameters $\lambda$ of the distortion model $\mathcal{T}$ can be computed for every target configuration $x$, i.e. if $\lambda=\lambda(x)$ is known. If sufficient information about the light sources producing the actual illumination in the environment is available (position and emission pattern of directional sources, ambient lighting, color and temperature, etc.) $\lambda(x)$ can be synthesized according to computer graphics rendering principles. Light source information is, however, very costly to obtain even in controlled environment, and practically not available in uncontrolled and dynamic environment. In the next section we investigate the problem of estimating $\lambda(x)$ from observations of moving people in unknown and dynamic environment.

Finally, if $\lambda(x)$ can be synthesized or estimated, the likelihood $f$ for particle filtering under non-uniform illumination can be modeled by $\log f(z \mid x, \lambda) \propto$ $D\left(z_{r}, \mathcal{T}(z \mid x, \lambda(x))\right)$ where $D$ is a suitable distance measure that considers similarity between the target reference pattern $z_{r}$ and the $\mathcal{T}$-corrected actual observation $z$. In our experiments we use Bhattacharyya distance between color histograms (see Sec. 4).

\section{Learning the Scene Illumination Using Walking People as Illumination Probes}

Given a reference pattern $z_{r}$ of an object, and an observation $\bar{z}$ of it at known location $\bar{x}$, we can compute the illumination parameters in that location by minimization of $D$, i.e. $\lambda(\bar{x})=\arg \min _{\lambda} D\left(z_{r}, \mathcal{T}(\bar{z} \mid \bar{x}, \lambda)\right)$. A possibility to approximate $\lambda(x)$ is therefore to collect a sufficient number of $\bar{x}, \bar{z}$-samples, determine $\lambda(\bar{x})$ on those samples, and compute $\lambda(x)$ from them e.g. by interpolation. In controlled 
environment this can be done in a calibration step, by placing a calibration target at some predefined locations distributed all around the environment and extracting associated observations from captured images using camera calibration and shape information of the calibration object. This, however, requires on-site manual intervention, and collecting a new set of dense $\bar{x}, \bar{z}$-samples for re-calibrating $\lambda(\bar{x})$ whenever illumination conditions change.

A more practical approach is then to use scene elements as calibration probes, and in our case, people. We use the multi-view person detector in [9] to sample $\lambda(x)$ as people move around in the environment. 9] uses a simple 3-dimensional shape model to detect and locate walking people using image motion extracted from multiple calibrated views. Differential measurements such as image motion used by the detector are largely invariant to illumination, leading to a sampling process that is conveniently uncorrelated with the estimation process for which we use it. The detector provides ground positions and associated bounding boxes of people in the various views, i.e. all the information needed to collect $\bar{x}, \bar{z}$ samples in an unsupervised and non-intrusive way.

\subsection{Hybrid Graphical Model with Bounded Memory Budget}

This sampling process can, however, not be controlled. People move around freely in uncontrolled environment, and illumination samples obtained this way are non-uniformly distributed both in space and across time. They are also affected by noise due to the difficulties faced by people detection in the presence of occlusions and clutter. In addition to this, not all samples can be considered for estimation since their number is unbounded as time grows, and a strategy is required to maintain essential information with a limited memory budget. Altogether, this calls for a probabilistic framework for online learning.

To develop such framework in this paper, we define a hybrid graphical model over the latent space $\lambda$, that is continuously updated as new samples are provided by the detector. The model is composed of a set of probe nodes $\bar{x}_{j}$ with associated observations $\bar{z}_{j}$ that are the most recent detections, and of a set of virtual, unobserved nodes at positions $x_{i}$ that we call support nodes. All nodes are connected in our model 1 , and the relations between their latent variables are defined pair-wise, through an MRF potential $\phi$ that imposes spatial smoothness. Probe nodes $\bar{x}_{j}$ do have observations $\bar{z}_{j}$, and we use the tracking likelihood $\phi\left(\lambda_{j}\right)=f\left(\bar{z}_{j} \mid \bar{x}_{j}, \lambda_{j}\right)$ defined in the previous section to assign likelihoods to latent variables $\lambda_{j}$ at node $\bar{x}_{j}$. Support nodes $x_{i}$, instead, do not have observations, and we want to learn their $\lambda_{i}$-likelihoods through 'likelihood transfer' from probe nodes, in a way that the same information is kept by the network if probe nodes are removed after transfer. Indeed, $\lambda_{i}$-likelihoods form the persistent and bounded memory budget of the network, while probe nodes with associated observations are connected only temporarily, in order to update the likelihoods at the support nodes with fresh information.

1 Though, for improved computational efficiency, the network can be made sparse by cutting dependencies among node pairs whose distance exceeds a threshold 

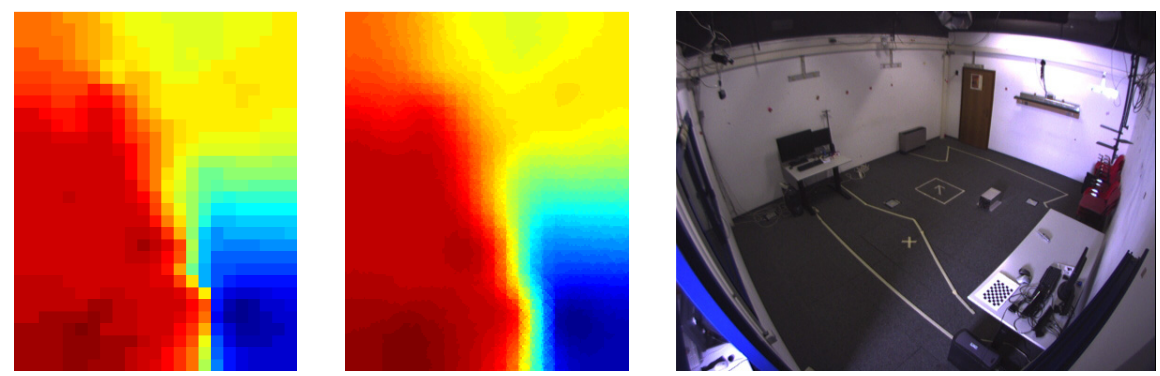

Fig. 2. MAP estimates on support nodes (left) and dense $\lambda$-lookup (mid) for the 1-dimensional von Kries model in Sec. 4, scenario sharp-illumination-edge

\subsection{Online Learning with the Hybrid Graphical Model}

The computational scheme that implements this idea is a two-step iterative procedure. In the first step we use standard sum-product message passing inference [10] on the hybrid graph to compute the beliefs at the support nodes. After convergence, we remove the probe nodes from the network, which now has different structure and no more observation. I.e., since probe messages are no longer available, all the information carried by the probe samples is now encoded in the support beliefs. We then conceptually 'freeze' the beliefs at the support nodes, and try to change the support likelihoods in a way that a new run of message passing on the persistent part of the network would produce the same, or close as possible, support beliefs. This second step requires a reformulation of standard message passing, where the roles of beliefs (now part of the model) and likelihoods (now subject to inference) are interchanged. Standard message passing, as used in the first step, iterates upon auxiliary variables $\mu$ (the messages) until convergence

$$
\mu_{k i}^{(1)}\left(\lambda_{i}\right) \leftarrow \int_{\lambda_{k}} \phi_{i k}\left(\lambda_{i}, \lambda_{k}\right) \phi_{k}\left(\lambda_{k}\right) \prod_{m \neq i} \mu_{m k}^{(1)}\left(\lambda_{k}\right)
$$

after which beliefs are read-out for each node $b_{i}\left(\lambda_{i}\right)=\phi_{i}\left(\lambda_{i}\right) \prod_{k} \mu_{k i}^{(1)}\left(\lambda_{i}\right)$. By substituting $\phi_{k}$ in Eq. 1 with its expression derived from the belief read-out equation we obtain the message iterations for the second step

$$
\mu_{k i}^{(2)}\left(\lambda_{i}\right) \leftarrow \int_{\lambda_{k}} \phi_{i k}\left(\lambda_{i}, \lambda_{k}\right) b_{k}\left(\lambda_{k}\right) / \mu_{i k}^{(2)}\left(\lambda_{k}\right)
$$

with likelihood read-out $\phi_{i}\left(\lambda_{i}\right) \leftarrow b_{i}\left(\lambda_{i}\right) / \prod_{k} \mu_{k i}^{(2)}\left(\lambda_{i}\right)$. Eq. 2 defines a new message passing scheme for online learning on hybrid graphical model with fixed memory budget, which is outlined in Alg.1, first part.

\subsection{Sampling $\lambda$ Using the Hybrid Graphical Model}

To find the solution to our original problem of estimating $\lambda(x)$ on random ground locations $x$ which we now name particles, we use again a hybrid graph: this time, 
while new set of probe observations available do

Step 0: Update the model;

add probe nodes and compute probe likelihoods;

update connectivity and potentials;

Step 1: Compute beliefs at support nodes;

iterate eq 1 till convergence;

read-out support beliefs;

remove probe nodes;

Step 2: Update likelihoods at support nodes;

iterate eq 2 till convergence;

read-out support likelihoods;

Step 3: Release updated model to lookup procedure;

end

while $\lambda$-values to be evaluated at some locations (particles) do

Step 0: Update the model;

eventually, update the model with a copy as released at Step 3 above;

add particles nodes and assign uniform likelihoods;

update connectivity (directional) and potentials;

Step 1: Compute beliefs at particles nodes;

compute particles node messages eq 1 ;

read-out particles node beliefs;

remove particles nodes;

Step 2: Return MAP estimates on particle nodes;

end

Algorithm 1. Online learning scheme (first) and $\lambda$-lookup procedure (second). Both can run in parallel, e.g. $\lambda$-lookup can be used for particle filter based people tracking while new detections are provided for updating the model, as in our experiments.

it is composed of the support node network with likelihoods and messages computed by the online learning scheme above, and a set of particle nodes with uniform likelihoods $\phi_{x}\left(\lambda_{x}\right) \equiv 1$ are added. Different from above, connectivity for particle nodes is directional: they are not allowed to pass messages to support nodes, to guarantee that the support network and all its variables are not modified by the addition of particle nodes (indeed, particle nodes are added for the only purpose of looking-up $\lambda$-values at the particle locations $x$ ). Through standard message passing we then obtain particle beliefs $b_{x}\left(\lambda_{x}\right)$ at $x$ and their MAP estimates $\lambda(x) \leftarrow \arg \max _{\lambda_{x}} b_{x}\left(\lambda_{x}\right)$ are returned. This procedure is outlined in Alg.1, second part.

This $\lambda$-lookup procedure is consistent: if we add a particle node with $x \equiv x_{i}$ for some support node $x_{i}$, we have $\mu_{i x}\left(\lambda_{x}\right)=b_{i}\left(\lambda_{i}\right) / \phi_{i}\left(x_{i}\right)$ from Eq. 1. and thus $b_{x}\left(\lambda_{x}\right)=b_{i}^{2}\left(\lambda_{i}\right) / \phi_{i}\left(x_{i}\right)$ whose MAP estimate should, qualitatively, be equivalent to that of $b_{i}\left(\lambda_{i}\right)$. I.e., looking-up $\lambda$ at $x \equiv x_{i}$ this way will return a value that is close to the MAP estimate of the support node $x_{i}$ itself. This can be verified with the example in Fig. 2. 


\section{Experiments}

In this section we report on the application of the proposed online learning framework to color-based tracking under non-uniform illumination. We start with an overview of the overall system, and then present results on data from two challenging scenarios.

The multi-view people detector in 9 is applied to data from each recorded scenario to collect illumination probes of a person walking across the environment, and the scene illumination is learnt from them independently for each view with the method in Sec. 3. The reference pattern $z_{r}$ is selected from the first 20 detection: 2 . The illumination model $\mathcal{T}$ is a one-dimensional von Kries linear model [11, i.e., $\lambda$ is a scalar parameter. Others [7] have used parameter vector to represent variability for each color channel ( $\lambda$ is three-dimensional in that case): in Fig. 3 we show that the illumination conditions in the recorded scenarios lie on a curve and thus are well described with a single scalar parameter, leading to significant savings in computation time. The distance measure $D$ defining the likelihood model $f(z \mid x, \lambda)$ (see Sec.2) is the Batthacharrya distance between quantized RGB color histograms (8 levels per channel for a total of 512 bins) of the reference pattern $z_{r}$ and the $\mathcal{T}$-corrected obseration $z$ extracted from the image using state ( $x$ is provided by the detector) and calibration information.

For tracking we use an extension of the multi-view people tracker in [12. It implements color based particle filtering as described in Sec. 2 with the likelihood above, and uses the lookup procedure in Sec. 3 to obtain the illumination parameter $\lambda(x)$ for each particle to compute color likelihoods.

We estimate the illumination conditions on two challenging scenarios, and give qualitative results and quantitative results in terms of tracking precision, comparing tracking performance with and without using such information. The data was recorded using four synchronized angled-view cameras $(512 \times 384$ jpeg @ $15 \mathrm{~Hz}$ ) installed in the corner of a $5 \times 6 \mathrm{~m}$ laboratory. The dataset, including manually labeled ground truth and a video showing our results, is available for download here http://tev.fbk. eu/DATABASES/VIPT.html

\subsection{Scenario 1 Sharp-Illumination-Edge}

For the first test sequence we created highly non-uniform illumination conditions using a Balcar Fluxlite illuminator as a directional light source. The illuminator was placed outside the room close to the entrance door, at a height of about $2.5 \mathrm{~m}$. It can be observed in Fig. 1 that a strong beam of light enters the room creating a sharp illumination edge across the room, much like direct sunlight entering from a window. Under these lighing conditions, we recorded a 3min test sequence showing a person moving around the environment.

Fig. 4 shows histograms of tracking error (distance from manually labeled ground truth) on the test sequence. Plot (a) shows that, even if all four views are used, color based tracking without illumination information as estimated with

\footnotetext{
${ }^{2}$ We choose the one with the 'richest' histogram, i.e. the one with the highest entropy.
} 


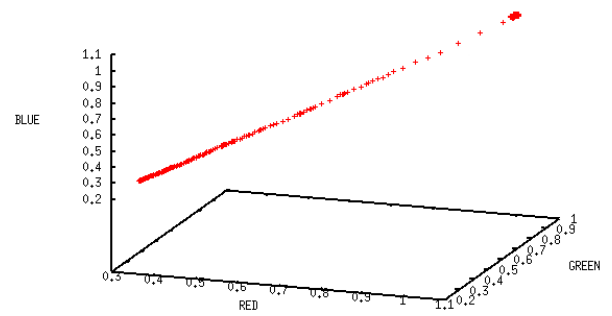

Fig. 3. Illumination parameter samples of the full-diagonal von Kries model. We acquired images of a color plate under the full range of illumination conditions in the two recorded scenarios, and computed the 3-dimensional parameter vectors using the $\lambda$-minimizer of $D$ in [11] (as in the calibration step in Sec. 2). It can be seen that under the observed illumination conditions a scalar illumination parameter suffices.
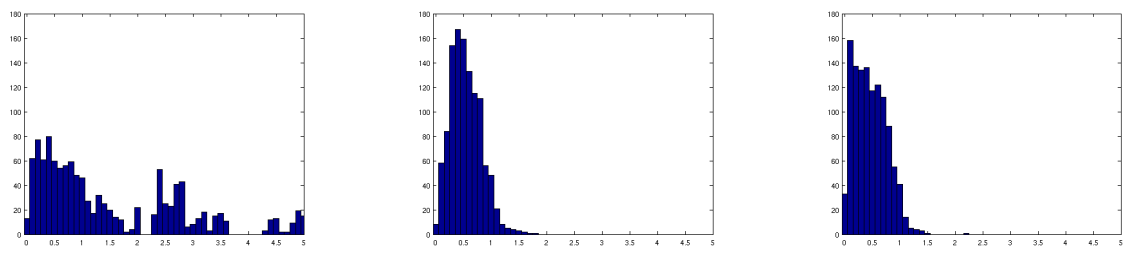

Fig. 4. Tracking precision in Scenario 1 sharp-illumination-edge using four cameras without illumination information (a), and with illumination information as estimated by the proposed online method (b) using one camera (see Fig. 2 for the scene as seen by the camera, and the estimated illumination conditions), (c) adding a second camera

the method proposed in this work breaks under such conditions. Instead, if such information is automatically estimated (Sec.3, Fig. 2) and properly integrated (Sec.2), good results can be obtained even with one (the most challenging among the four as it is directed along the light beam) view only (b), while precision further increases if another camera is added (c).

\subsection{Scenario 2 Changing-Illumination}

This scenario is designed to show that the proposed framework is suitable for online adaptation under changing illumination conditions. We move the illuminator behind a window of the lab, and after some time we smoothly turn the light to change the orientation of the illumination beam it generates while the person is moving inside the room. After that, other three people enter the room to make the tracking on the final part of the sequence even more challenging.

Fig. 5 shows how illumination information is incrementally learnt by our method. We use 20 subsequent detections at each iteration in Alg. 1 for online 

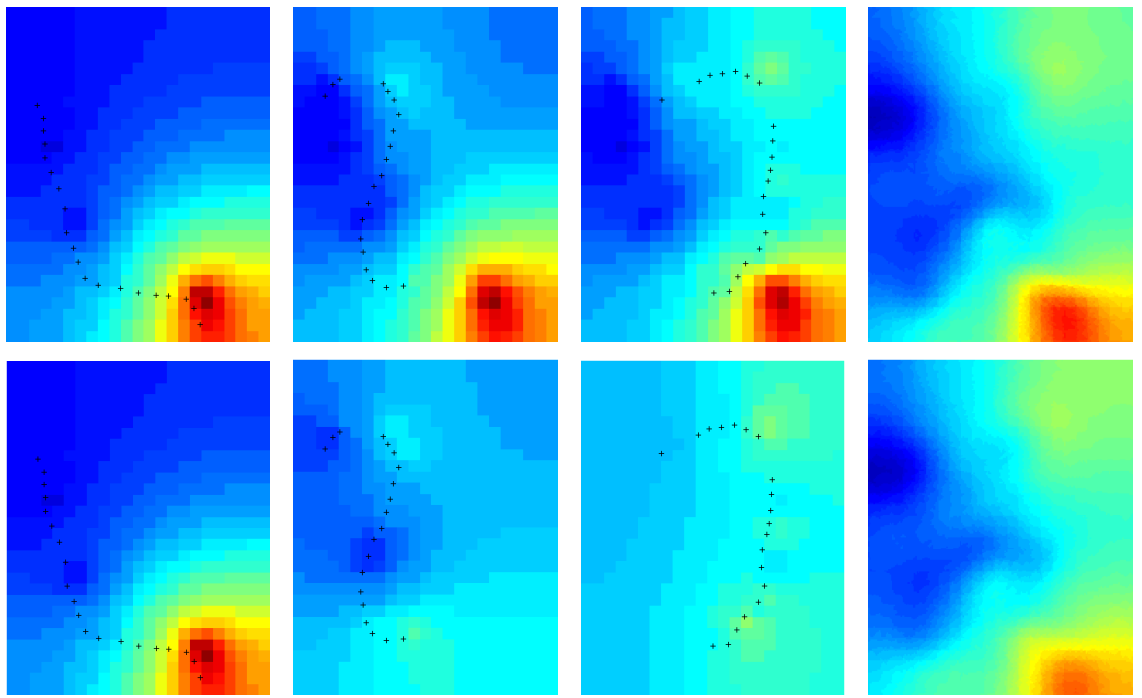

Fig. 5. Online learning on Scenario 2 changing-illumination. Top: MAP estimates on support nodes after first three updates $(\mathrm{a}, \mathrm{b}, \mathrm{c})$ and final dense $\lambda$-lookup (d). Crosses denote detections, i.e. locations of probe observations, used for the update. Bottom: first tre updates $(e, f, g)$ using standard message passing on the same hybrid network, and (h) $\lambda$-lookup of the batch network (all probes attached) for comparison with (d).
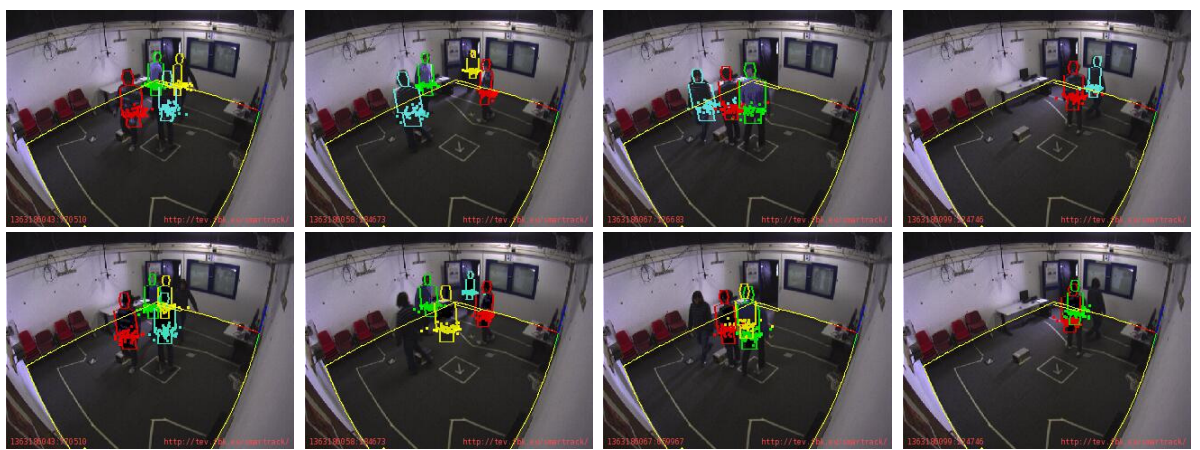

Fig. 6. Color based tracking in Scenario 2 changing-illumination with (first row) and without (second) illumination information as estimated by the proposed online method

learning. The positions of those probe nodes displayed as crosses in Fig. 5 show the path of the person across the room, and it can be observed how the scene illumination information is shaped along it, and remains unchanged elsewhere. I.e., illumination information sensed dynamically from the scene by observing the person walking around is persistently transferred into the parameters of the hybrid graphical network, i.e., into the virtual likelihoods of the un-observed support nodes. Standard message passing without our inventive step in Eq. 2 is 
not able to incrementally learn, as the only information maintained is encoded in the messages which readily diffuses into the network once probe nodes are removed (see Fig. 5 bottom row). Finally, Fig. 6] shows screenshots of our realtime tracking on the final part of the sequence.

\section{Conclusions}

We presented a novel framework for online learning with hybrid graphical model composed of a dynamic set of observed nodes and a fixed budget of virtual nodes whose likelhoods are the parameters of the network constituting its persistent memory. Parameters are updated by information transfer from each new set of observed nodes through a novel message passing scheme. We applied this framework to estimate the scene illumination for robust color based tracking under non-uniform and time varying illumination. Future work will be on developing a nore solid theory for the novel scheme, and on exploring other applications where the proposed framework can advantageously be applied.

\section{References}

1. Yilmaz, A., Javed, O., Shah, M.: Object tracking: A survey. ACM Comput. Surv. 38 (2006)

2. Cristani, M., Farenzena, M., Bloisi, D., Murino, V.: Background subtraction for automated multisensor surveillance: a comprehensive review. Eurasip. J. Adv. Signal Process. 43 (2010)

3. Vezzani, R., Grana, C., Cucchiara, R.: Probabilistic people tracking with appearance models and occlusion classification: The AD-HOC system. Pattern Recogn. Lett. 32, 6 (2011)

4. Fleet, D.J., El-Maraghi, T.F.: Robust online appearance models for visual tracking. IEEE Trans. Patt. Anal. Mach. Intell. 25, 10 (2003)

5. Tsai, Y.P., Ko, C.H., Hung, Y.P., Shih, Z.C.: Background removal of multiview images by learning shape priors. IEEE Trans. Image Proc. 16, 10 (2007)

6. Camplani, M., Luis Salgado, L.: Adaptive background modeling in multicamera system for real-time object detection. SPIE Optical Eng. 50, 12 (2011)

7. Zen, G., Lanz, O., Messelodi, S., Ricci, E.: Tracking Multiple People with Illumination Maps. In: Proc. ICPR (2010)

8. Bardet, F., Chateau, T., Ramadasan, D.: Illumination aware MCMC Particle Filter for long-term outdoor multi-object simultaneous tracking and classification. In: Proc. ICCV (2009)

9. Lanz, O., Messelodi, S.: A Sampling Algorithm for Occlusion Robust Multi Target Detection. In: Proc. AVSS (2009)

10. Yedidia, J.S., Freeman, W.T., Weiss, Y.: Understanding Belief Propagation and Its Generalizations. MERL tech. rep. (2003)

11. Lecca, M., Messelodi, S.: Linking the von Kries model to Wien's law for the estimation of an illuminant invariant image. Pattern Recogn. Lett. 32(15) (2011)

12. Lanz, O.: Approximate bayesian multibody tracking. IEEE Trans. Patt. Anal. Mach. Intell. 28(9) (2006) 